Presented at the 1997 IESNA Annual Conference and submitted to the proceedings.

\title{
Simulating the Visual Performance of Electrochromic Glazing for Solar Control
}

\author{
Charles Ehrlich \\ Building Technologies Department \\ Environmental Energy Technologies Division \\ Ernest Orlando Lawrence Berkeley National Laboratory \\ University of California \\ 1 Cyclotron Road \\ Berkeley, California 94720 USA
}

February 1997

This work was supported by the Assistant Secretary for Energy Efficiency and Renewable Energy, Office of Building Technology, Building Technologies Program, of the U.S. Department of Energy under Contract No. DEAC03-76SF00098. 


\section{Introduction}

A new technology called electrochromic glazing promises to provide the building industry with a means to dynamically control the visual appearance and solar gain of windows. Electrochromic glazing is a technology which allows an otherwise ordinary looking piece of laminated glass to change tint with the application of a small electrical charge. Prototype electrochromic devices have been produced in sizes up to one square foot, however, manufacturers are several years away from producing glazing samples large enough to fill the aperture of a typical perimeter office. Tooling up for the production of large samples is prohibitively expensive unless some assurance of the marketability of these new electrochromic products can be demonstrated.

Electrochromic glazings defy traditional performance rating mechanisms because of the temporal dimension of their thermal and visual characteristics. Every electrochromic glazing assembly has an infinite number of states at which the thermal and visual appearance could be characterized. Furthermore, the rate at which the assembly changes between states is vital for a complete understanding.

Work of the International Energy Agency, Annex 18, Task A2/A3 ${ }^{1}$ includes advancements in whole building thermal performance simulation techniques. Likewise, a simulation technique which allows manufacturers to answer questions about the visual performance (visual appearance through time) of their electrochromic glazing products would provide further insight into the ability of the technology to control the detrimental effects of solar exposure and if shown to be successful, hasten the product into the marketplace. The simulation technique presented herein provides a method for animating a virtual environment which stimulates the visual cortex of a viewer of the animation on a video screen in a manner similar to the experience of an occupant of the actual space. Although no video screen can reproduce the 10,000 to 1 dynamic range which is experienced in the built environment, a new software algorithm takes advantage of the strengths and weaknesses of the human eye to optimize the dynamic range of the video screen. The animated sequence created using this technique is intended to provide 
glazing researchers and manufacturers with a tool to better understand the human impact of electrochromic glazing technology.

\section{Assumptions}

The Electrochromic Glazing Office Animation demonstrates the visual performance of a typical office equipped with current state-of-the art, electrochromic glazing. The visual phenomena which an occupant might experience are faithfully reproduced on your video screen by the rendering power of the Radiance Synthetic Imaging System ${ }^{2}$. This video simulates the ability of the electrochromic glazing to respond to direct solar exposure through west-facing glazing. The visual appearance and switching rate are measured from one foot square samples of prototype electrochromic devices and are applied directly to the animation. No attempt is made to maintain constant workplane illuminance levels (the most likely goal of electrochromic glazing installations), nor is any attempt made to simulate actual weather data. These other scenarios and the animation of faster switching technologies are the subject of future research.

This method assumes that the sources of illumination (sun and computer monitor) do not change position throughout the animation sequence. Although the sun would have moved approximately 5 degrees during the span of this 20 minute animation sequence, its movement is not the focus of the simulation and does not significantly change the intensity of the solar exposure. This method takes advantage of the principal of the scalability of light to avoid the significant time involved in calculating separate Radiance renderings for each combination of sky condition (direct sun versus no direct sun) and electrochromic glazing transmittance. In other words, the amount of light in the office is directly proportional to the visible transmittance of the electrochromic glazing. The angular dependent transmittance characteristics of typical float glass are included in the calculations.

The subject room is a ten feet wide by 30 feet deep by 10 feet tall, perimeter glazed office. The glazing spans the width of the office and reaches from 30 inches above the floor at the window sill to the ceiling plane. The wall reflectance is $57 \%$, the ceiling reflectance is $76 \%$ and the floor reflectance is $22 \%$. The transmittance of the 
electrochromic glazing varies between $74 \%$ and $3.2 \%$. The sky distribution is created with the Radiance gensky command which conforms to the luminance distribution of the CIE Clear Sky ${ }^{3}$.

\section{Method}

The image compositing technique used to create the Electrochromic Glazing Office Animation employs separate images of the office generated with only one source of illumination, added together in variable percentages to come up with the final image. First, an image of the office with only the computer monitor "turned on" and without direct sun and without ambient sky component is rendered. This results in a very dark room indeed. (See figure 1.) Then, an image of the office with only the ambient sky component is rendered (figure 2). Next, an image of the office with only the direct sun component is rendered (figure 3). Each of these images is rendered with exactly the same viewing parameters resulting in three images that are perfectly aligned, pixel by pixel. All three of these component images take approximately 2 hours to render on a $100 \mathrm{MHz}$ Pentium based computer. 

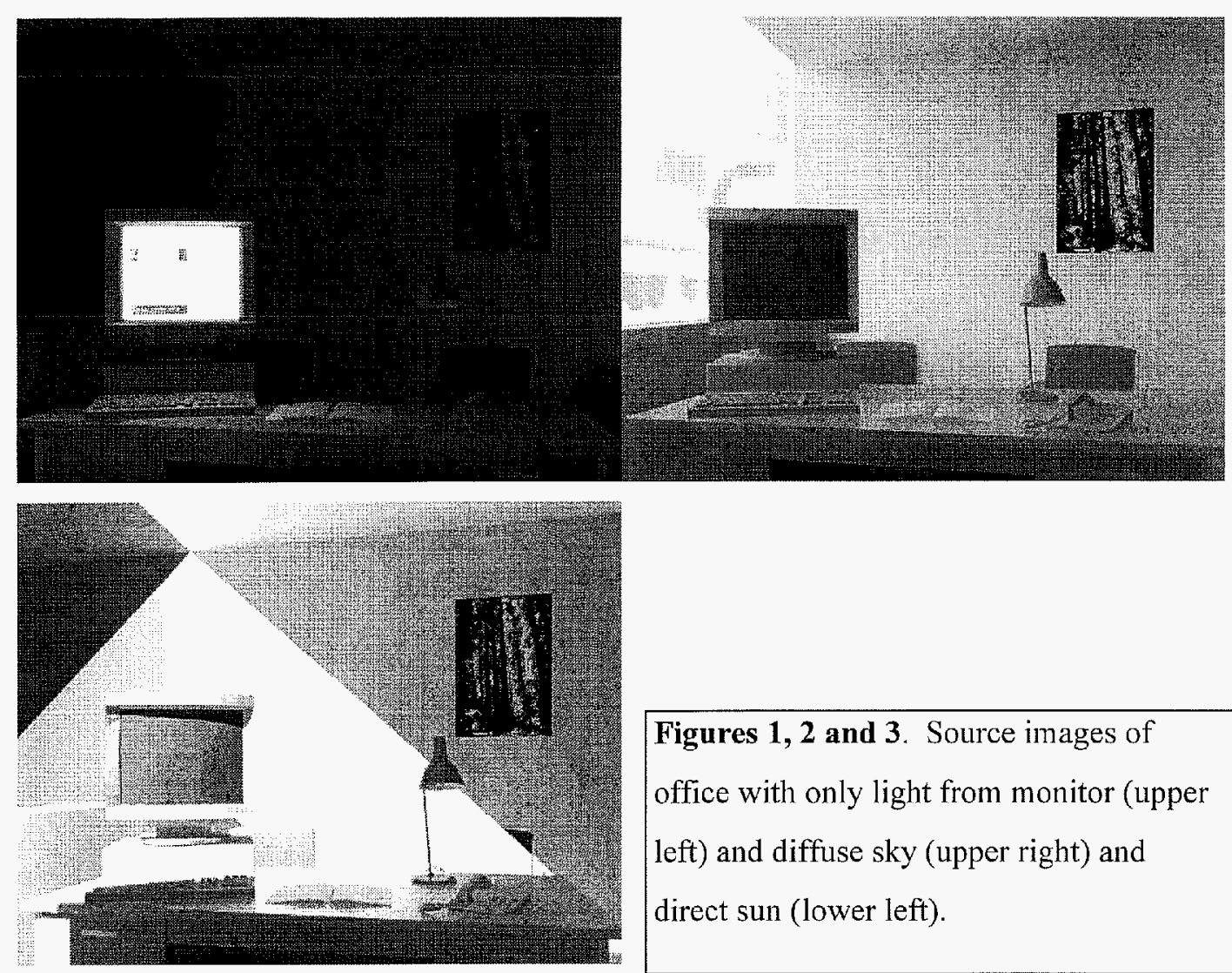

Figures 1, 2 and 3. Source images of

office with only light from monitor (upper left) and diffuse sky (upper right) and direct sun (lower left).

Then, the Radiance pcomb program adds together each pixel of the images according to a function which defines the percentage of each light source which is desired. The desired percentage depends partly upon the measured transmittance of the electrochromic device in response to a maximal coloration or bleaching charge, and partly upon the behavior of an imaginary, non-diffusing, completely opaque cloud which occludes the solar source at the desired moment (the percentage of direct sun component is ramped down to zero). 


\begin{tabular}{|ll|}
\hline 1.10 & 77.49880 \\
1.13 & 77.47570 \\
1.16 & 77.27250 \\
1.20 & 74.62451 \\
1.23 & 71.42550 \\
1.26 & 68.11131 \\
1.30 & 65.01461 \\
1.33 & 62.18341 \\
1.36 & 59.55110 \\
1.40 & 57.13971 \\
1.43 & 54.93630 \\
1.46 & 52.86730 \\
1.50 & 50.99120 \\
1.53 & 49.20560 \\
1.56 & 47.60501 \\
& $\ldots$ \\
\hline
\end{tabular}

Table 1 (above). Time sequence transmittance at $555 \mathrm{~nm}$.

Table 2 (right). Spectral transmittance from $780 \mathrm{~nm}$ to $380 \mathrm{~nm}$ (actual data measured at $5 \mathrm{~nm}$ interval.)

\begin{tabular}{|ll|}
\hline 780.00 & 1.18500 \\
760.00 & 1.22721 \\
740.00 & 1.27630 \\
720.00 & 1.35561 \\
700.00 & 1.49361 \\
680.00 & 1.63001 \\
660.00 & 1.76500 \\
640.00 & 1.91971 \\
620.00 & 2.11620 \\
600.00 & 2.34921 \\
580.00 & 2.56300 \\
560.00 & 2.86810 \\
540.00 & 3.48931 \\
520.00 & 4.18801 \\
500.00 & 4.87141 \\
480.00 & 5.58380 \\
460.00 & 6.04020 \\
440.00 & 6.32670 \\
420.00 & 6.31000 \\
400.00 & 6.14831 \\
380.00 & 5.83011 \\
\hline
\end{tabular}

The author performed time sequence measurements at $555 \mathrm{~nm}$ and full spectrum measurements of large prototype (one foot square) electrochromic devices provided by leading glazing industry manufacturers. These measurements employed a Lambda 19 spectrophotometer equipped with an integrating sphere to obtain the transmittance and front and back reflectance of the electrochromic devices. Time sequence transmittance and reflectance at $555 \mathrm{~nm}$ are correlated to the photopically weighted, average visible transmittance. Spectral measurements are converted to XYZ tristimulus values and then to Radiance RGB values using the Radiance cie_xyx.cal and xyz2rgb.cal ${ }^{4}$ functions respectively. These functions replicate the CIE chromaticity mapping functions in the native Radiance procedural language.

The time sequence and average RGB transmittance of the electrochromic device are plugged into an ASCII control file (See Table 3). The control file includes instructions on how to change the sky conditions and the image overlay text. The control file then informs the image compositing process through linear interpolation such that changing sky conditions and electrochromic coloration are accurately simulated. The interior ambient and direct solar lighting conditions change in proportion to the visible transmittance of the electrochromic glazing. 


\begin{tabular}{|c|c|c|c|c|c|c|c|c|c|}
\hline reel time & time min & Tvis & $T$ red & T green & $\mathrm{T}$ blue & volts & etime & sun & Overlay Text \\
\hline 0 & 0 & 75.4 & 0.743496 & 0.786286 & 0.458545 & 0 & -1 & 0 & \\
\hline 0.05 & 0.05 & 75.4 & 0.586785 & 0.687204 & 0.435461 & 0 & 0 & .00002 & \\
\hline 0.1 & 0.1 & 75.4 & 0.586785 & 0.687204 & 0.435461 & 0 & 0 & .005 & \\
\hline 0.2 & 0.2 & 75.4 & 0.586785 & 0.687204 & 0.435461 & 0 & 0 & .02 & \\
\hline 0.33 & 0.33 & 64.4 & 0.443953 & 0.588145 & 0.410906 & 1.3 & 0.23 & 1 & \\
\hline 0.45 & 0.45 & 53.8 & 0.297868 & 0.471181 & 0.376255 & 1.3 & 0.35 & 1 & \\
\hline 0.68 & 0.68 & 41.9 & 0.184056 & 0.362366 & 0.336961 & 1.3 & 0.58 & 1 & \\
\hline 1.05 & 1.05 & 31.3 & 0.099895 & 0.259232 & 0.289305 & 1.3 & 0.95 & 1 & \\
\hline 1.74 & 1.74 & 21.9 & 0.048882 & 0.176925 & 0.240767 & 1.3 & 1.64 & 1 & \\
\hline 2.85 & 2.85 & 14.7 & 0.022404 & 0.117226 & 0.195179 & 1.3 & 2.75 & 1 & \\
\hline 4.6 & 4.6 & 9.7 & 0.010049 & 0.076900 & 0.155451 & 1.3 & 4.5 & 1 & Time Lapse 10X \\
\hline 4.7 & 7.62 & 6.4 & 0.005203 & 0.054353 & 0.127372 & 1.3 & 7.52 & 1 & Time Lapse 10X \\
\hline 5 & 17.6 & 4.6 & 0.002605 & 0.036307 & 0.100092 & 1.3 & 16.48 & 1 & Time Lapse 10X \\
\hline 5.1 & 17.7 & 3.2 & 0.002605 & 0.036307 & 0.100092 & 1.3 & 16.48 & 1 & Full Coloration in 17 minutes \\
\hline 5.15 & 17.8 & 3.2 & 0.002605 & 0.036307 & 0.100092 & 1.3 & 16.48 & .02 & Full Coloration in 17 minutes \\
\hline 5.2 & 17.8 & 3.2 & 0.002605 & 0.036307 & 0.100092 & 1.3 & 16.48 & .005 & \\
\hline 5.25 & 17.8 & 3.2 & 0.002605 & 0.036307 & 0.100092 & 1.3 & 16.48 & .00002 & \\
\hline 5.3 & 17.9 & 4.6 & 0.005203 & 0.054353 & 0.127372 & -0.3 & 0.1 & 0 & \\
\hline 5.43 & 18.03 & 6.4 & 0.010049 & 0.076900 & 0.155451 & -0.3 & 0.23 & 0 & \\
\hline 5.58 & 18.18 & 9.7 & 0.022404 & 0.117226 & 0.195179 & -0.3 & 0.38 & 0 & \\
\hline 5.88 & 18.48 & 14.7 & 0.048882 & 0.176925 & 0.240767 & -0.3 & 0.68 & 0 & \\
\hline 6.29 & 18.89 & 21.9 & 0.099895 & 0.259232 & 0.289305 & -0.3 & 1.09 & 0 & \\
\hline 6.72 & 19.32 & 31.3 & 0.184056 & 0.362366 & 0.336961 & -0.3 & 1.52 & 0 & \\
\hline 7.15 & 19.75 & 41.9 & 0.297868 & 0.471181 & 0.376255 & -0.3 & 1.95 & 0 & \\
\hline 7.62 & 20.22 & 53.8 & 0.443953 & 0.588145 & 0.410906 & -0.3 & 2.42 & 0 & \\
\hline 8.05 & 20.65 & 64.4 & 0.586785 & 0.687204 & 0.435461 & -0.3 & 2.85 & 0 & \\
\hline 9.7 & 22.3 & 75.4 & 0.743496 & 0.786286 & 0.458545 & 0 & 4.5 & 0 & Full Bleaching in 4.5 minutes \\
\hline 9.8 & 22.3 & 75.4 & 0.743496 & 0.786286 & 0.458545 & 0 & 4.5 & 0 & \\
\hline
\end{tabular}

Table 3. Animation control file.

Finally, each composited rendering is then passed through a Radiance pixel conditioning program called pcond ${ }^{5}$ which first determines an appropriate exposure function for that image and then applies a dynamic, pixel-by-pixel, exposure compensation accordingly. The assumption is that the electrochromic glazing is changing slowly enough for the human eye to immediately adapt to the changing lighting levels. The exposure level is based upon a foveal-weighted luminance scan and a luminance histogram of every pixel of the image. The histogram is used by pcond to generate a custom brightness mapping function for each frame of the animation. The mapping function uses the understanding that the human eye is sensitive to local changes in luminance, but insensitive to global luminance differences. Luminance values of the computed luminance image are mapped to optomize the dynamic range of the video screen output pixel brightnesses.

Each frame of the animation sequence is created in this manner and then sent frame by frame to a Sony laser video disk recorder. All of the composited frames for a 10 minute animation at 1 frame per second take approximately 2 hours to calculate on a 100 
$\mathrm{MHz}$ Pentium based computer. Individual VHS tapes are then produced from the video disk master.

Because there is no audio track, table 4 may help to understand what is happening while viewing the video animation sequence. The animation may be viewed via the World Wide Web at: http://radsite.lbl.gov/electro/visual/ec_m3.mpg or the author may be contacted for a copy of the animation on VHS tape. If viewing via the Web, be sure to set your MPEG playback software to play the sequence back at 1 frame per second.

1. 15:00:00, sun obscured by a cloud, glass at full transmittance: Tvis $=74 \%$

2. 15:00:03, cloud moves away and direct sun enters the office

3. 15:00:12, electrochromic glazing begins to color at its maximum rate

4. 15:04:06 through 15:17:42, video is in 10X time lapse to prevent boredom

5. $15: 17: 42$, electrochromic glazing reaches its maximum coloration: Tvis $=3.2 \%$

6. 15:17:48, the cloud moves in front of sun obscuring all direct sun

7. 15:17:54, electrochromic glazing begins to bleach at its maximum rate

8. 15:22:18, electrochromic glazing reaches its maximum transmittance: Tvis $=74 \%$

Table 4. Video animation sequence highlights.

\section{8:22:18 Tris $=75.4 \%$}

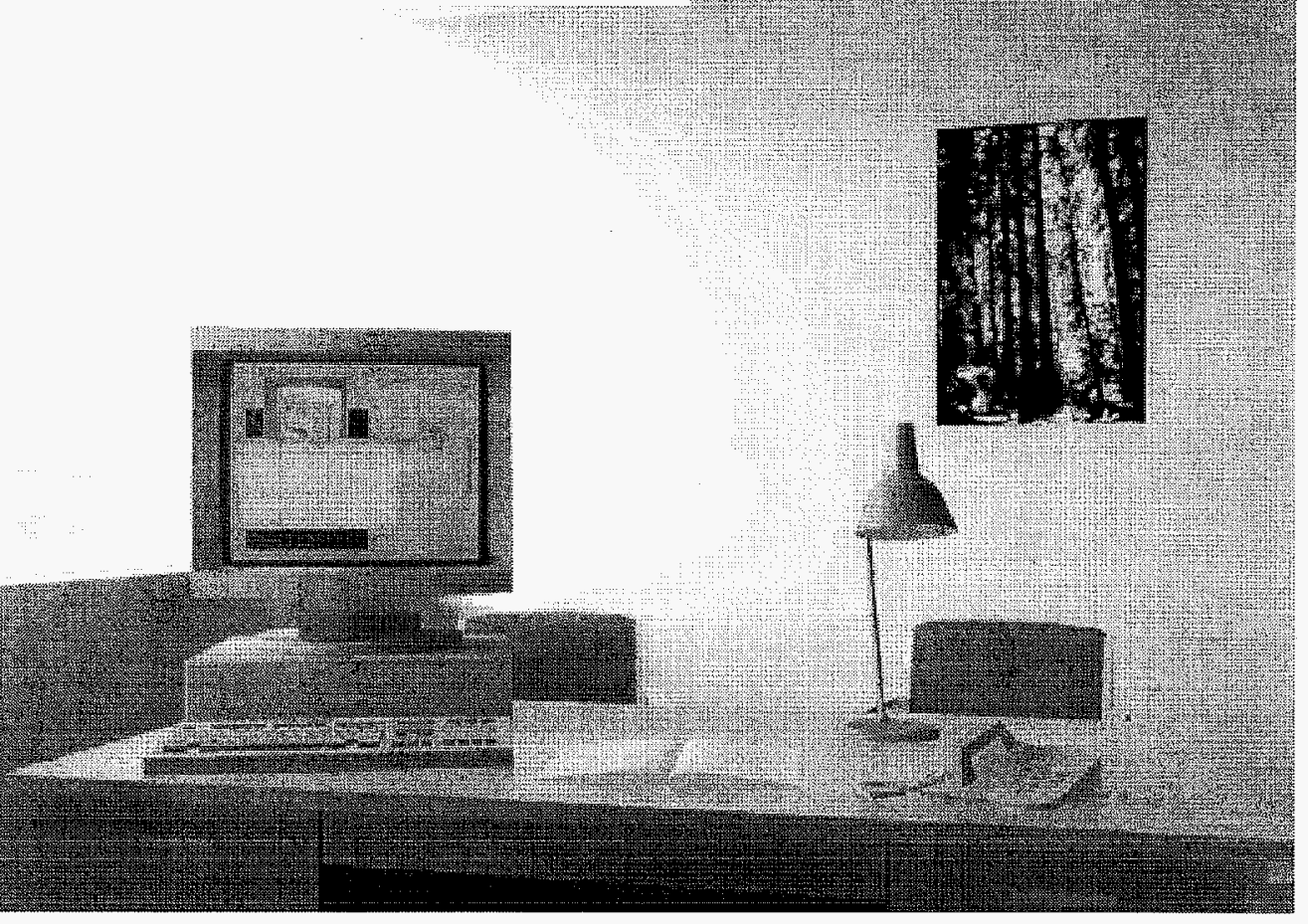

Figure 4. One example frame of the animation sequence showing the combined effect of light from the direct sun, diffuse sky and monitor. 


\section{Conclusions}

Although difficult to present the results of an animation simulation with paper and ink media, it is hoped that the author has conveyed both the substance of the method and sense for the degree of realism of the results. The physical measurements of the electrochromic device properties when combined with the latest rendering simulation methods which account for the dynamic exposure characteristics of the human eye can be used to produce a new tool for use in marketing studies and product development research. The technique presented here is well suited for demonstrating products that have characteristics that change over time.

Final results from the use of this animation in office worker surveys are not yet available, however this animation shows that the switching rates are too slow for office occupants, and even at the minimum transmittance of $3.2 \%$, glare from west facing glazing in the afternoon is still great enough to require additional solar control devices such as traditional venetion blinds. 
${ }^{1}$ International Energy Agency, Annex 18, Task A2/A3. Internal working document, T18/A2A3/FPR/97.

${ }^{2}$ The Radiance Lighting Simulation and Rendering System, Gregory J. Ward, Computer Graphics Proceedings, Annual Conference Series, 1994.

${ }^{3}$ CIE (1972), Standardization of Luminance Distribution on Clear Skies, Commission Internationale de l'Eclarage, CIE 22-1972.

${ }^{4}$ CIE (1932), Commission Internationale de l'Eclarage. Proceedings, 1931.

${ }^{5}$ A Visibility Matching Tone Reproduction Operator for High Dynamic Range Scenes, Gregory J. Ward, Holly Rushmeier, and Christine Piatko, Submitted to Computer

Graphics Proceedings Annual Conference Series, 1997.

\section{Acknowledgements}

This work was supported by the Assistant Secretary for Energy Efficiency and Renewable Energy, Office of Building Technology, State and Community Programs (BTS), Office of Building Systems of the U.S. Department of Energy under Contract No. DE-AC03-76SF00098. 